\title{
Unidirectional pulmonary airflow patterns in the savannah monitor lizard
}

\section{Citation}

Schachner, Emma R., Robert L. Cieri, James P. Butler, and C. G. Farmer. 2013. “Unidirectional Pulmonary Airflow Patterns in the Savannah Monitor Lizard." Nature 506 (7488) (December 11): 367-370. doi:10.1038/nature12871.

\section{Published Version}

doi:10.1038/nature12871

\section{Permanent link}

http://nrs.harvard.edu/urn-3:HUL.InstRepos:32631102

\section{Terms of Use}

This article was downloaded from Harvard University's DASH repository, and is made available under the terms and conditions applicable to Other Posted Material, as set forth at http:// nrs.harvard.edu/urn-3:HUL.InstRepos:dash.current.terms-of-use\#LAA

\section{Share Your Story}

The Harvard community has made this article openly available.

Please share how this access benefits you. Submit a story.

Accessibility 


\title{
Unidirectional pulmonary airflow patterns in the savannah monitor lizard
}

\author{
Emma R. Schachner, ${ }^{1}$ Robert L. Cieri, ${ }^{1}$ James P. Butler, ${ }^{2,3}$ CG Farmer ${ }^{1}$
}

The unidirectional airflow patterns in the lungs of birds have long been considered a unique and specialized trait associated with the oxygen demands of their volant lifestyle, endothermic metabolism ${ }^{1}$ and unusual pulmonary architecture ${ }^{2,3}$; however, the discovery of similar flow patterns in the lungs of crocodilians indicates that this character is likely ancestral for all archosaurs, the group that includes extant birds and crocodilians as well as their extinct relatives, such as pterosaurs and dinosaurs ${ }^{4-6}$. Unidirectional flow in birds results from aerodynamic valves, rather than from sphincters or other physical mechanisms ${ }^{7,8}$, and similar aerodynamic valves appear to be at work in crocodilians ${ }^{4-6}$. Due to anatomical and developmental similarities in the primary and secondary bronchi of birds and crocodilians, these structures and airflow patterns may be homologous ${ }^{4-6,9}$. Thus the origin of this pattern is at least as old as the split between crocodilians and birds, which occurred in the Triassic ${ }^{10}$; however, it is possible that this pattern of flow is even older. This constitutes an alternative hypothesis, which can be tested by investigating patterns of airflow in members of the outgroup to birds and crocodilians, the Lepidosauromorpha (tuatara, lizards, and snakes). Here we show region-specific unidirectional airflow in the lungs of the savannah monitor lizard (Varanus exanthematicus). The presence of unidirectional flow in the lungs of $V$. exanthematicus thus gives rise to two possible evolutionary scenarios: (1) unidirectional airflow evolved independently in archosaurs and monitor lizards, or (2) these flow patterns are homologous in archosaurs and $V$. exanthematicus, having evolved only once in ancestral diapsids. If unidirectional airflow is plesiomorphic for Diapsida, this respiratory character can be reconstructed for extinct diapsids, and evolved in a small ectothermic tetrapod during the Paleozoic Era at least 100 million years before the origin of birds.

The lungs of lepidosaurs have been assumed to be ventilated tidally, an idea based on their bronchial architecture ${ }^{11}$; however, direct measurements of flow are lacking. Furthermore, fluid dynamics are often non-intuitive, and phenomena such as Venturi effects can result in complicated patterns of flow. It is important to characterize patterns of flow in lizards to assess the evolutionary history of the vertebrate lung. Varanids (73 species) are a widely distributed group of anguimorph lizards ${ }^{12}$ with the oldest unambiguous fossil appearance of Varanus from the Upper Eocene and Lower Oligocene of Egypt ${ }^{13}$. The external morphology of the genus Varanus is superficially conservative; however, they vary in mass by almost five orders of magnitude and occupy a range of ecological niches (semi-aquatic to arboreal) ${ }^{14,15}$. Compared to other lepidosaurs, varanids have high aerobic capacities, with Varanus caudolineatus having one of the highest rates of oxygen consumption ever recorded in a non-avian reptile ${ }^{16}$. High gas exchange capacities of varanids arise in part from their ability to supplement costal breathing with gular pumping ${ }^{17}$. These lizards possess multichambered (i.e., multibronchial) lungs (Fig. 1a-e), which have long been used as a phylogenetic character for Varanoidea ${ }^{18}$, and thus varanid pulmonary anatomy has received considerable attention ${ }^{18-22}$.

Varanid lungs are large, structurally asymmetrical and multichambered ${ }^{11,20}$ (SI video 1) (Fig. 1a-c). The dorsal surface is firmly attached to the ribs along the majority of 
their length $^{21}$. The primary bronchus runs the length of the lung, ballooning into a large sac-like bronchus just distal to the ostium of the caudal-most lateral bronchus (Fig. 1b; As). The general arrangement of the secondary bronchi arising from the intrapulmonary primary bronchus follows what appears to be a stereotyped branching pattern. Without corroborating developmental data to support this observation, suppositions of bronchial identity remain tentative. Nevertheless, the anatomy of the adult bronchial tree can be visualized from CT data of individual specimens of $V$. exanthematicus. The first bronchus to arise from the primary bronchus (the cervical bronchus, $\mathrm{Cb}$ ) has a cartilaginous tube shaped ostium that immediately makes a hairpin turn, running cranially and expanding into a large tubular bronchus terminating at the apex of the lung (Fig. 1c; Cb). This bronchus is anatomically reminiscent of the cervical ventral bronchus in crocodilians ${ }^{6,9}$. Arising sequentially from the lateral surface of the primary bronchus is a series of 9-11 variably shaped large sac-like bronchi, termed lateral bronchi (Fig. 1c; L1-10). Lateral bronchi communicate with the adjacent bronchi through numerous small intercameral perforations. A similar series of sequentially arranged sac-like bronchi arise off of the medial surface of the primary bronchus from small ostia and run caudomedially, rotating to a ventromedial position. Depending upon the individual, either the first or second medial bronchus on (usually) the right lung extends cranially along the ventral surface of the lung, terminating just distal to the carina. This bronchus is much smaller in the left lung and does not extend cranially. Along the dorsal surface of the primary bronchus in both lungs, small sac-like bronchi emerge in an asymmetrical pattern (Fig. 1b; Ssb). The respiratory parenchyma is largely restricted to the central and craniodorsal regions of the lung, with the saccular regions positioned at the cranial tip and caudoventral areas ${ }^{21}$. Small tertiary bronchi extend towards the pleural surface, forming hexagonal faveolar parenchyma $^{11,21}$.

The airflow patterns in $V$. exanthematicus are heterogeneous, with tidal and unidirectional flow observed in different regions of the lungs. Unidirectional airflow was measured using heated thermistor flow meters in vivo $(\mathrm{n}=5)$ and in excised lungs $(\mathrm{n}=9)$ in the caudal-most, large lateral bronchus (generally L10 depending upon the individual) (Fig. 1c, d; 2a-c). Flow was also observed by visualizing the movements of microspheres and/or pollen suspended in water in excised lungs ( $n=5$; SI video 2$)$. Tidal flow was recorded in a cranial lateral bronchus (excised, $n=2$ ), while unidirectional flow was observed visually in the rest of the lateral bronchi (L2-L10; $n=4)$ and the cervical bronchus $(\mathrm{n}=4)$. Biased flow (i.e., a significantly stronger magnitude of flow present during only one phase of the respiratory cycle) was measured in the abdominal sac-like bronchus (with thermistors ( $n=4)$ and microspheres $(n=4)$ in excised lungs), with air arriving dorsally via the primary bronchus during inspiration, travelling caudoventrally to the caudal surfaces of the last lateral bronchus and through the intercameral perforations along the shared bronchial walls during expiration (Fig. 1e). Flow between the lateral bronchi is interbronchial unidirectional flow, meaning that the flow travels in the same direction from one bronchus to another during both phases of the respiratory cycle.

These observations thus constitute evidence supporting the conclusion that the global pattern of flow in the bronchial tree of $V$. exanthematicus is predominantly unidirectional, composed of cranial and caudal regions of unidirectional airflow (Fig. 1e). Flow in the cervical bronchus appears to maintain its one-way direction by jetting in association with the anatomy of the ostium and proximally constricted bronchus, coupled 
with connections to small tertiary bronchi (Fig. 1b; Tb). Flow between the lateral bronchi is likely also maintained via jetting, in conjunction with the branching angle of each individual bronchus relative to the primary bronchus and the proximal constriction of each bronchus, thus constituting aerodynamic valving. The flow between bronchi is possible because of intercameral perforations, much like those found in archosaur lungs despite the differences in their respective bronchial architecture. Aerodynamic valves arise from the geometry and branching angles of the primary and secondary bronchi, with valving mediated by the convective momentum of gas flow in this particular geometry. We suggest that this is the mechanism biasing flow in one direction in the lung of $V$. exanthematicus due to the absence of any physical flaps or muscular sphincters within their bronchial tree, and because flow patterns were unchanged in excised lungs. Thus it appears that the flow arises from the same aerodynamic phenomena seen in the archosaurian lung ${ }^{7}$.

Unidirectional flow patterns have been measured in both avian and crocodilian lungs, indicating that this trait is likely plesiomorphic for Archosauria. The presence of unidirectional flow in regions of the lung of Varanus exanthematicus thus raises two hypotheses reflecting different evolutionary scenarios (Fig. 3). The first hypothesis is that unidirectional flow patterns evolved independently in both Archosauria and Lepidosauria, and are a convergent apomorphy of both groups. The alternative hypothesis is that this pulmonary character is homologous in archosaurs and $V$. exanthematicus, having evolved only once, and is thus the ancestral state for diapsids (Lepidosauria + Archosauria). Relative to other lepidosaurs, varanids are particularly derived with a significantly more complex bronchial arrangement than their more basal relatives (see Fig. 3) ${ }^{11,23}$. The structure of varanid airways is different from that of archosaurs, both in terms of bronchial geometry as well as its branching sequence along the primary bronchus, making it difficult to favour one evolutionary scenario over the other. The presence of archosaur-like aerodynamic valves and unidirectional flow in the varanid lung suggests that this trait evolved in ancestral diapsids; however, differences in the patterns of flow between varanids (caudal to cranial in the ventrolateral sac-like bronchi) and archosaurs (caudal to cranial in the dorsal tube-shaped secondary bronchi) coupled with significant differences in the arrangement of the secondary bronchi, can only be interpreted as convergent until the airflow patterns in more basal lepidosaurs are measured (Fig. 3). Due to the considerable amount of variability in the lepidosaurian respiratory system, both across the entire clade as well as within individual groups, it will be essential to investigate flow patterns in multiple representative species from each major group (e.g., Sphenodon, Iguania, Gekkota, Scincomorpha and other non-varanid anguimorphs) to shed light on this question (Fig. 3).

Determining when unidirectional airflow patterns first evolved has implications for understanding both the origin and function of respiratory patterns in non-avian reptiles, as well as reconstructing lung physiology and morphology in extinct taxa. If demonstrated to be ancestral for Diapsida, unidirectional airflow patterns can be parsimoniously reconstructed in all extinct diapsids. If these flow patterns evolved convergently between varanids and archosaurs, then this would suggest that the ability to ventilate unidirectionally holds an adaptive significance to these taxa.

\section{Methods Summary}


In vivo data were collected from five live specimens of Varanus exanthematicus (mass 350g-930g), and ex vivo data from ten specimens. Animals were acquired from the California Zoological Supply (live) and donated (deceased) from James Dix, Utah's Reptile Rescue Service. All experiments were performed in accordance with and approved by the University of Utah IACUC. Three individuals were CT scanned at 100 $\mathrm{kVp} 400 \mathrm{Ma}(0.6 \times 0.4 \mathrm{~mm})$. Digital models of the bronchial tree were segmented in Avizo 7.1. Measurements of airflow were made with dual heated thermistor airflow probes surgically implanted into individual bronchi. The probes were connected to an HEC 132C Thermistor Flowmeter (Hector Engineering Co., Inc., Elletsville, IN). The analogue output was converted to a digital signal (Biopac Systems Inc, Goleta, CA) and recorded on a computer using AcqKnowledge software (Biopac Systems Inc., Goleta, $\mathrm{CA}$ ). Airflow at the nares was measured with a pneumotach (Hans Rudolph Inc., Shawnee, KS). Flow traces in live animals were recorded during natural breathing; traces measured in excised lungs were acquired from artificial ventilation (60 cc syringe). Video of the movement of saline containing microspheres $(222 \mu \mathrm{m}$ diameter, Thermo Scientific) and pollen through excised lungs was taken with a Canon EOS T2i (1080p). The raw CT data are available from the Dryad Digital Repository: http://doi.org/10.5061/dryad.v1d30.

1 Maina, J. N. Development, structure, and function of a novel respiratory organ, the lung-air sac system of birds: to go where no other vertebrate has gone. Biological Reviews 81, 545-579 (2006).

2 Brackenbury, J. H. Lung-air-sac anatomy and respiratory pressures in the bird. Journal of Experimental Biology 57, 543-550 (1972).

3 Maina, J. N. Spectacularly robust! Tensegrity principle explains the mechanical strength of the avian lung. Respiration Physiology \& Neurobiology 155, 1-10 (2006).

4 Farmer, C. G. The provenance of alveolar and parabronchial lungs: insights from paleoecology and the discovery of cardiogenic, unidirectional airflow in the American alligator (Alligator mississippiensis). Physiological and Biochemical Zoology 83, 561-575 (2010).

5 Farmer, C. G. \& Sanders, K. Unidirectional airflow in the lungs of alligators. Science 327, 338-340 (2010).

6 Schachner, E. R., Hutchinson, J. R. \& Farmer, C. G. Pulmonary anatomy in the Nile crocodile and the evolution of unidirectional airflow in Archosauria. PeerJ, DOI 10.7717/peerj.7760 (2013).

7 Butler, J. P., Banzett, R. B. \& Fredberg, J. J. Inspiratory valving in avian bronchi: aerodynamic considerations. Respiration Physiology 72, 241-256 (1988).

8 Hazelhoff, E. H. Structure and function of the lung of birds. Poultry Science 30, 3-10 (1951).

9 Sanders, R. K. \& Farmer, C. G. The pulmonary anatomy of Alligator mississippiensis and its similarity to the avian respiratory system. The Anatomical Record 295, 699-714 (2012). 
10 Nesbitt, S. J. The early evolution of archosaurs: relationships and the origin of major clades`. Bulletin American Museum of Natural History 352, 1-292 (2011).

11 Perry, S. F. in Biology of the Reptilia Vol. 19 (Morphology G) Contribu. Herpetol. vol 14 (eds C. Gans \& A. S. Gaunt) 1-92 (Society for the Study of Amphibians and Reptiles, 1998).

12 Conrad, J. L., Balcarcel, A. M. \& Mehling, C. M. Earliest example of a giant monitor lizard (Varanus, Varanidae, Squamata) PLoS ONE 7, e41767 (2012).

13 Holmes, R. B., Murray, A. M., Attia, Y. S., Simons, E. L. \& Chatrath, P. Oldest known Varanus (Squamata: Varanidae) from the Upper Eocene and Lower Oligocene of Egypt: support for an African origin of the genus. Palaeontology 53, 1099-1110 (2010).

14 Collar, D. C., Schulte II, J. A. \& Losos, J. B. Evolution of extreme body size disparity in monitor lizards (Varanus). Evolution 65, 2664-2680 (2011).

15 Pianka, E. R. Evolution of body size: varanid lizards as a model system. The American Naturalist 146, 398-414 (1995).

16 Thompson, G. G. \& Withers, P. C. Standard and maximal metabolic rates of goannas (Squamata: Varanidae). Physiological Zoology 70, 307-323 (1997).

17 Owerkowicz, T., Farmer, C. G., Hicks, J. W. \& Brainerd, E. L. Contribution of the gular pump to ventilation. Science 284, 1661-1663 (1999).

18 Becker, H.-O., Böhme, W. \& Perry, S. F. Die Lungenmorphologie der Warane (Reptilia: Varanidae) und ihre systematisch-stammesgeschichtliche Bedeutung. Bonner zoologische Beiträge 40, 27-56 (1989).

19 Burnell, A., Collins, S. \& Young, B. A. The postpulmonary septum of Varanus salvator and its implication for Mosasaurian ventilation and physiology.

Bulletin de la Societe Geologique de France 183, 159-169 (2012).

20 Kirschfeld, U. Eine bauplananalyse der waranlunge. Zoologische Beitraege NF 16, 401-440 (1970).

21 Maina, J. N., Maloiy, G. M. O., Warui, C. N., Njogu, E. K. \& Kokwaro, E. D. Scanning electron microscope study of the morphology of the reptilian lung: the savanna monitor lizard Varanus exanthematicus and the Pancake Tortoise Malacochersus tornieri. The Anatomical Record 224, 514-522 (1989).

22 Perry, S. F. \& Duncker, H. R. Lung architecture, volume and static mechanics in five species of lizards. Respiration Physiology 34, 61-81 (1978).

23 Wallach, V. in Biology of the Reptilia Vol. 19 (eds C. Gans \& A.S. Gaunt) Ch. 2, 93-295 (Society for the Study of Amphibians and Reptiles, 1998).

24 Milani, A. Beiträge zur Kenntniss der Reptilienlunge. . Zoologische Jahrbücher 7, 545-592 (1894).

25 Milani, A. Beiträge zur Kenntnis der Reptilienlunge. II. Zoologische Jahrbücher 10, 93-156 (1897).

26 Milsom, W. K. \& Vitalis, T. Z. Pulmonary mechanics and the work of breathing in the lizard, Gekko gecko. Journal of Experimental Biology 113, 187-202 (1984).

Supplementary Information is available in the online version of the paper. 
Acknowledgements We thank J. Dix (Reptile Rescue Service) for donation of deceased varanid specimens, J. Bourke for assistance with Avizo, and D. Shafer for German translations. This work was supported by an American Association of Anatomists Postdoctoral Fellowship and an American Philosophical Society Franklin Research Grant to E.R.S, National Science Foundation Grants to C.G.F. (IOS-1055080 and IOS0818973) and a generous donation to the Farmer lab by Sharon Meyer.

Author Contributions E.R.S and R.L.C. conducted the in vivo surgeries. All authors collected data on excised lungs. E.R.S. acquired the CT scans and generated the 3D digital models. C.G.F. and J.P.B. supervised and contributed ideas throughout the project. All authors contributed to the manuscript.

\author{
Author Information \\ ${ }^{1}$ Department of Biology, University of Utah, Salt Lake City, UT 84112 \\ ${ }^{2}$ Division of Sleep Medicine, Dept. Medicine, Harvard Medical School, Boston, MA \\ ${ }^{3}$ Molecular and Integrative Physiologic Science Program, Dept. Environmental Health, \\ Harvard School of Public Health, Boston, MA 02115
}

Correspondence and requests for materials should be addressed to E.R.S. (eschachner@gmail.com) or C.G.Farmer (cg.frmr@gmail.com). Reprints and permissions information are available at www.nature.com/reprints. The authors declare no competing financial interests.

\title{
Figure legends
}

1. Pulmonary anatomy and airflow patterns of Varanus exanthematicus.

(a): Volume rendered skeleton and segmented lungs. (b): Solid representation of the bronchial tree. Tb, tertiary bronchi; Ssb, secondary sac-like bronchi; As, abdominal saclike bronchus. (c): Same as (b) with tertiary and medial bronchi removed. $\mathrm{Cb}$, cervical bronchus; L1-L10, lateral bronchi 1-10. (d): Bronchi in which flow was measured in excised lungs (orange/grey), and in vivo in this animal (pink). (e): A diagram of the lung with arrows showing the direction of measured airflow (L5, 7, 9-10, and abdominal sac). Paired large and small arrows: biased flow, blue arrows: interbronchial flow.

\section{Airflow recorded in vivo.}

(a): Left lateral view of the left excised and inflated lung of Varanus exanthematicus. Arrow indicates where the airflow probe was surgically implanted for all in vivo measurements. Numbers represent regions where flow was recorded in excised lungs: 1, cervical bronchus (excised: $n=2$; saline: $n=4$ ); 2 , last lateral bronchus (in vivo: $n=5$; excised: $n=9$; saline: $n=4$ ); 3 , abdominal sac (excised: $n=4$, saline: $n=4$ ). Scale bar $=1 \mathrm{~cm}$. (b): Tidal airflow measured at the nares. Grey regions: inhalation, unshaded regions: exhalation. (c): Largely unidirectional flow in L10; flows indicate directionality, not actual magnitudes. 
3. Phylogeny for Diapsida showing lungs of representative taxa.

Greyscale images are modified from Milani ${ }^{24,25}$ and transected. The coloured 3D images are the bronchial tree (right lateral view). Images not to scale. (a) Diapsida. (b) Sphenodon punctatus. (c) Crocodile sp. (left) and Alligator mississippiensis (right). (d) Squamata. (e) Iguana iguana (left) and Polychrus marmoratus (right). (f) Gekko gecko ${ }^{26}$. (g) Lacerta viridis. (h) Python sp. in dorsal view ${ }^{23}$ (i) Varanus bengalensis (left) and $V$. exanthematicus (right). The blue regions of the phylogeny reflect the hypothesis that unidirectional airflow evolved convergently; the green arrow shows the alternate hypothesis of an ancestral origin.

\section{Full Methods}

In vivo data were collected from five live specimens of Varanus exanthematicus (Mass $350 \mathrm{~g}-930 \mathrm{~g}$ ). Data were collected from the excised lungs of ten specimens of mixed sex and unknown age. All of the live varanids were acquired from the California Zoological Supply and the deceased specimens were donated to the University of Utah by James Dix (Utah's Reptile Rescue Service). All experiments were performed in accordance with and approved by the University of Utah IACUC. Three individuals were CT scanned at 100 $\mathrm{kVp} 400 \mathrm{Ma}(0.6 \times 0.4 \mathrm{~mm})$. Digital models of the bronchial tree, lung surface and skeleton were segmented by hand in Avizo 7.1 (http://www.vsg3d.com/avizo/standard) using a Wacom Intuos 4 pen tablet. The images were edited into figures in Adobe Photoshop CS6, and the 3D files exported from Avizo were edited into a video file in Adobe Premiere CS6. Measurements of airflow were made with dual heated thermistor airflow probes surgically implanted into individual bronchi of the lungs. The probes were connected to an HEC 132C Thermistor Flowmeter (Hector Engineering Co., Inc., Elletsville, IN). The analogue output was converted to a digital signal (Biopac Systems Inc., Goleta, CA) and recorded on a laptop using AcqKnowledge software (Biopac Systems Inc., Goleta, CA). Airflow at the nares was measured with a pneumotach (Hans Rudolph Inc., Shawnee, KS). Flow traces in live animals were recorded as they breathed hypercapnic gas; traces measured in excised lungs were acquired from artificial ventilation (60 cc syringe). Five of the smaller lungs were excised and filled with microphere (222 $\mu \mathrm{m}$ diameter, Thermo Scientific) infused saline and/or pollen grains. Video of movement of the microspheres was recorded with a Canon EOS T2i (1080p) digital camera. The raw CT data are available from the Dryad Digital Repository: http://doi.org/10.5061/dryad.v1d30. 


\section{a}
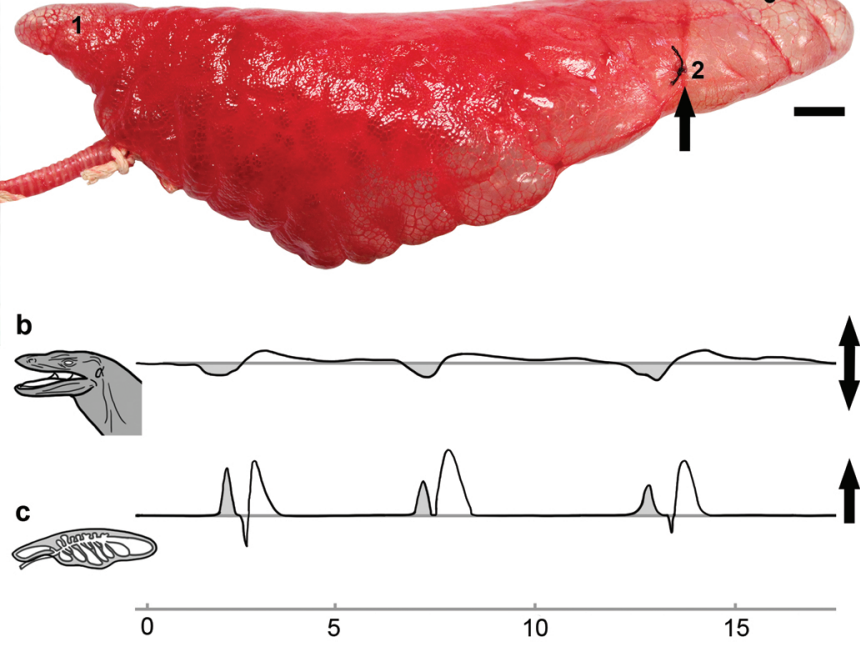

Time (seconds) 


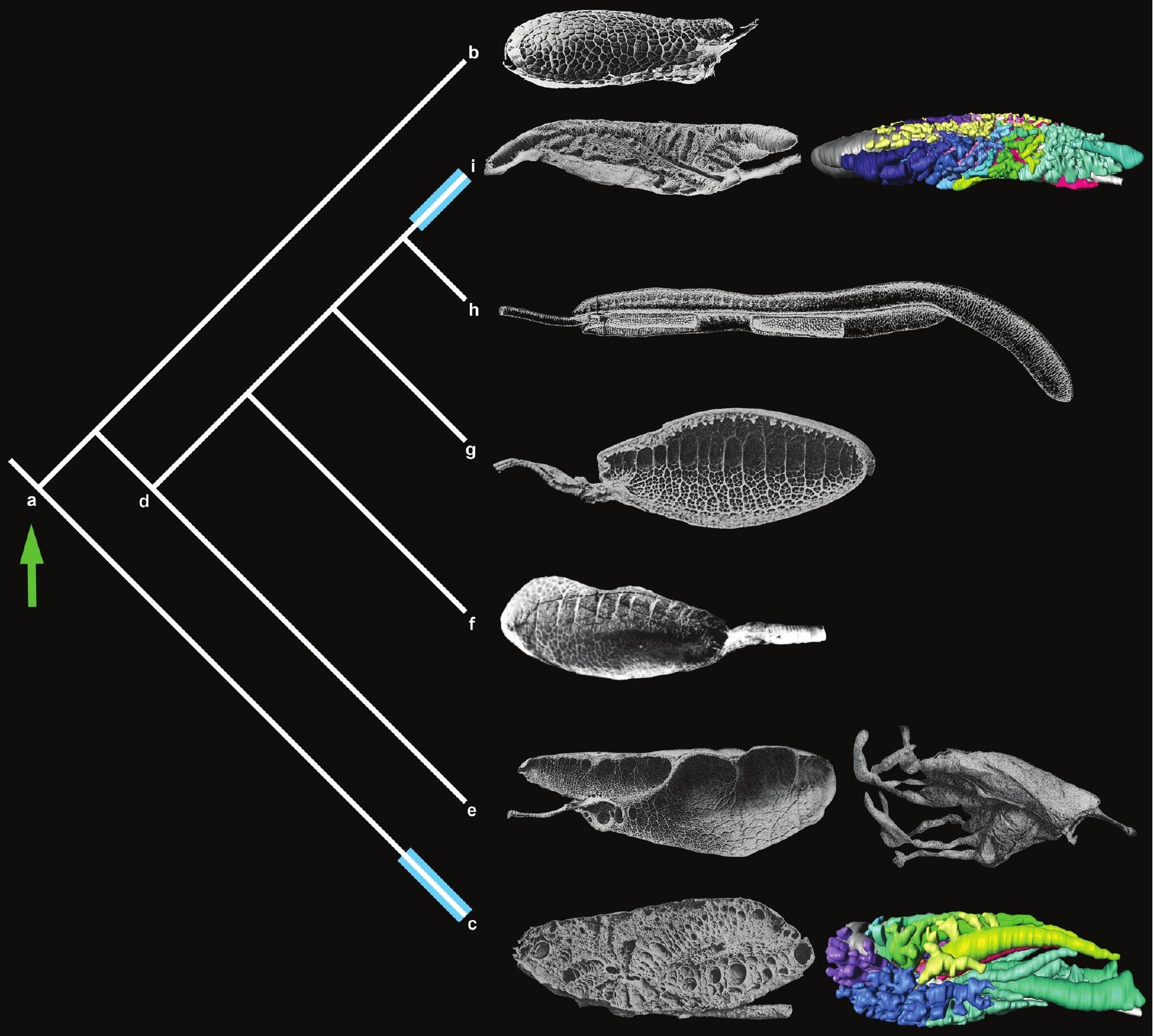

